\title{
Stability Properties of Linear Volterra Integrodifferential Equations in a Banach Space
}

\author{
By \\ Yoshiyuki Hino* and Satoru Murakami ${ }^{\dagger}$ \\ (Chiba University and Okayama University of Science, Japan) \\ Dedicated to Professor Toshiki Naito on his sixtieth birthday
}

\begin{abstract}
For linear Volterra integrodifferential equations, we characterize the uniform asymptotic stability property of the zero solution by a property for the resolvent operator. In particular, for equations of convolution type, we characterize the uniform asymptotic stability property in terms of the integrability of the resolvent operator, as well as the invertibility of the characteristic operator. Furthermore, we apply our results to nonhomogeneous equations with asymptotically almost periodic forcing terms, and establish some results on the existence of asymptotically almost periodic solutions.

Key Words and Phrases. Volterra integrodifferential equation, Stabilities, Resolvent operator, Asymptotically almost periodic solutions.

2000 Mathematics Subject Classification Numbers. Primary 34K14; Secondary $34 \mathrm{~K} 30$.
\end{abstract}

\section{Introduction}

In this paper we are concerned with the study of stability properties of linear Volterra integrodifferential equations

$$
\frac{d u(t)}{d t}=A u(t)+\int_{0}^{t} B(t, s) u(s) d s, \quad t \in \boldsymbol{R}^{+}:=[0, \infty),
$$

$\left(\mathrm{E}_{\infty}\right)$

$$
\begin{gathered}
\frac{d v(t)}{d t}=A v(t)+\int_{-\infty}^{t} B(t, s) v(s) d s, \quad t \in \boldsymbol{R}:=(-\infty, \infty), \\
\frac{d u(t)}{d t}=A u(t)+\int_{0}^{t} B(t, s) u(s) d s+p(t), \quad t \in \boldsymbol{R}^{+}
\end{gathered}
$$

and

\footnotetext{
* Partly supported by the Grant-in-Aid for Scientific Research (C), No. 16540139, of the Japanese Ministry of Education, Culture, Sports, Science and Technology.

$\dagger$ Partly supported by the Grant-in-Aid for Scientific Research (C), No. 16540177, of the Japanese Ministry of Education, Culture, Sports, Science and Technology.
} 
$\left(\mathbf{P}_{\infty}\right) \quad \frac{d v(t)}{d t}=A v(t)+\int_{-\infty}^{t} B(t, s) v(s) d s+p(t), \quad t \in \boldsymbol{R}$

where $A$ is the generator of a compact $C_{0}$ semigroup of bounded linear operators on a Banach space $\boldsymbol{X}$ and $B(t, s)$ is a bounded linear operator on $\boldsymbol{X}$ which satisfies the almost periodicity in $t$ uniformly for $s$, together with some conditions cited in Section 2. Volterra integrodifferential equations have been treated in several works; see, e.g., $[4,5,6,7,9,10,12,13,16,17,21,24]$ and the references therein. In case of $\operatorname{dim} \boldsymbol{X}<\infty$, the authors in [9, 10] treated linear Volterra integrodifferential equations, and established some equivalent relationships among several stability properties in Volterra integrodifferential equations $(E)$ and $\left(E_{\infty}\right)$. Furthermore, they obtained a result which characterizes the uniform asymptotic stability property in terms of the integrability of the resolvent for the equations, and applied it to ensure the existence of bounded solutions for the nonhomogeneous equation with bounded forcing terms. The purpose of this paper is to extend several results in $[9,10]$ to the equations with infinite dimensional $\boldsymbol{X}$. We emphasize that the method employed in [9, 10] for the equations with $\operatorname{dim} \boldsymbol{X}<\infty$ is not directly applicable to the analysis for the equations with infinite dimensional $\boldsymbol{X}$. Indeed, if one proceeds the way in $[9$, 10] for the analysis in case of $\operatorname{dim} \boldsymbol{X}=\infty$, there will arise a difficulty in the establishment of the integrability of the resolvent. To overcome this, in this paper we will focus attention on a property for the resolvent which is weaker than the integrability, and deduce that the total stability property in (E) is equivalent to the weaker property for the resolvent (Theorem 3.1). In fact, when $(\mathrm{E})$ is of convolution type, that is, $B(t, s) \equiv B(t-s)$, the weaker property yields the integrability of the resolvent; consequently, we can characterize the uniform asymptotic stability property in (E) in terms of the integrability of the resolvent operator, as well as the invertibility of the characteristic operator (Theorem 3.3). Thus, our Theorem 3.3 for linear Volterra integrodifferential equations of convolution type is a generalization of some results in [7, 16] for equations with infinite dimensional $\boldsymbol{X}$. Subsequently, by utilizing the weaker property for the resolvent, we will obtain some results on the existence of asymptotically almost periodic solutions for the nonhomogeneous equations with asymptotically almost periodic forcing terms (Theorem 5.1), together with an inclusion relation on the Bohr spectrum of the almost periodic part for asymptotically almost periodic solutions (Theorem 5.4). We point out that there are several works dealing with subjects similar to ours. For example, the authors in $[3,15]$ have treated evolutionary integral equations of convolution type which admit unbounded linear operators as kernel functions, and established some results on the asymptotic behavior of bounded solutions and the uniform stability property of resovent operators. Also, the authors in $[1,2,18]$ 
have treated evolution families which corresponds to the resolvent of nonautonomous linear differential equations in Banach spaces, and established some results on stability properties of the evolution families such as exponential stability, exponential expansiveness, and so on. For more informations on these studies, see $[3,15,1,2,18]$ and the references therein.

\section{Definitions and some preparatory results}

Throughout this paper, we will use the following notations. $\left(\boldsymbol{X},|\cdot|_{\boldsymbol{X}}\right)$ denotes the Banach space, $C(J ; \boldsymbol{X})$ denotes the space of all $\boldsymbol{X}$-valued continuous functions on $J, J \subset \boldsymbol{R}$, and $B C(J ; \boldsymbol{X})$ denotes the subspace of $C(J ; \boldsymbol{X})$ consisting of all bounded and continuous functions on $J$. Clearly, $B C(J ; X)$ is a Banach space equipped with the supremum norm, which we denote by $\|\cdot\|_{J}$. Also, $L(\boldsymbol{X})$ denotes the Banach space of all bounded linear operators on $\boldsymbol{X}$ equipped with the operator norm $\|\cdot\|$.

We now consider the linear Volterra integrodifferential equation

$$
\frac{d u(t)}{d t}=A u(t)+\int_{0}^{t} B(t, s) u(s) d s, \quad t \in \boldsymbol{R}^{+}
$$

where $A$ is the generator of a compact $C_{0}$ semigroup $\{T(t)\}_{t \geq 0}$ of bounded linear operators on a Banach space $\boldsymbol{X}$ and $B(t, s)$ is a bounded linear operator on $\boldsymbol{X}$, continuous for $-\infty<s \leq t<\infty$ with respect to the operator norm and almost periodic, where $B(t, s)$ is said to be almost periodic in $t$ uniformly for $s$, if for any $\varepsilon>0$ and any compact set $J_{0} \subset \boldsymbol{R}^{-}, \boldsymbol{R}^{-}=(-\infty, 0]$, there exists a positive number $l\left(\varepsilon, J_{0}\right)$ such that any interval of length $l\left(\varepsilon, J_{0}\right)$ contains a $\tau$ for which $\|B(t, t+s)-B(t+\tau, t+\tau+s)\|<\varepsilon$ for all $t \in \boldsymbol{R}$ and $s \in J_{0}$. For the properties of an almost periodic function with parameters, see [23].

From the almost periodicity of $B(t, s)$ it follows that $\{B(t, t+s) \mid t \in \boldsymbol{R}\}$ is a relatively compact subset of $L(\boldsymbol{X})$ for each $s \in \boldsymbol{R}^{-}$. Moreover, for any sequence $\left\{t_{k}^{\prime}\right\}$ in $\boldsymbol{R}$, there is a subsequence $\left\{t_{k}\right\}$ such that the sequence $\left\{B\left(t+t_{k}, t+t_{k}+s\right)\right\}$ converges to some $D(t, s)$ in $L(\boldsymbol{X})$ uniformly for $(t, s) \in$ $\boldsymbol{R} \times J_{0}$, where $J_{0} \subset \boldsymbol{R}^{-}$is any ompact interval. In this case, the function $C(t, s):=D(t, s-t)$ is continuous for $-\infty<s \leq t<\infty$ and almost periodic in $t$ uniformly for $s$. We call $C(t, s)$ a function in the hull of $B(t, s)$.

For any $(\sigma, \phi) \in \boldsymbol{R}^{+} \times B C([0, \sigma] ; \boldsymbol{X})$ and $p \in B C([\sigma, \infty) ; \boldsymbol{X})$, there exists a (unique) function $u: \boldsymbol{R}^{+} \mapsto \boldsymbol{X}$ such that $u$ is continuous on $[\sigma, \infty)$ and the following relation holds [8]:

$$
u(\theta)=\phi(\theta), \quad \theta \in[0, \sigma]
$$

and 


$$
u(t)=T(t-\sigma) \phi(\sigma)+\int_{\sigma}^{t} T(t-s)\left\{\int_{0}^{s} B(s, \tau) u(\tau) d \tau+p(s)\right\} d s, \quad t \geq \sigma .
$$

The function $u$ is called a (mild) solution of $(\mathrm{P})$ through $(\sigma, \phi)$ on $[\sigma, \infty)$, and denoted by $u(\cdot, \sigma, \phi ; p)$. Similarly, for any $(\sigma, \phi) \in \boldsymbol{R} \times B C((-\infty, \sigma] ; \boldsymbol{X})$ and $p \in B C([\sigma, \infty) ; \boldsymbol{X})$, there exists a (unique) function $v: \boldsymbol{R} \mapsto \boldsymbol{X}$ such that $v$ is continuous on $[\sigma, \infty)$ and the following relation holds [8]:

$$
v(\theta)=\phi(\theta), \quad \theta \in(-\infty, \sigma]
$$

and

$$
v(t)=T(t-\sigma) \phi(\sigma)+\int_{\sigma}^{t} T(t-s)\left\{\int_{-\infty}^{s} B(s, \tau) v(\tau) d \tau+p(s)\right\} d s, \quad t \geq \sigma .
$$

The function $v$ is called a (mild) solution of $\left(\mathrm{P}_{\infty}\right)$ through $(\sigma, \phi)$ on $[\sigma, \infty)$, and denoted by $v(\cdot, \sigma, \phi ; p)$.

Definition 2.1. The zero solution of $(\mathrm{E})\left(\operatorname{resp} .\left(\mathrm{E}_{\infty}\right)\right.$ ) is said to be total stable (in short, TS) if and only if for any $\varepsilon>0$, there exists a $\delta(\varepsilon)>0$ such that for any $(\sigma, \phi) \in \boldsymbol{R}^{+} \times B C([0, \sigma] ; \boldsymbol{X})(\operatorname{resp} . \quad(\sigma, \phi) \in \boldsymbol{R} \times B C((-\infty, \sigma] ; \boldsymbol{X}))$, and $p \in B C([\sigma, \infty) ; \boldsymbol{X})$, if $\|\phi\|_{[0, \sigma]}<\delta(\varepsilon)$ (resp. $\left.\|\phi\|_{(-\infty, \sigma]}<\delta(\varepsilon)\right)$ and $\|p\|_{[\sigma, \infty)}<\delta(\varepsilon)$, where $\|\phi\|_{[0, \sigma]}=\sup _{s \in[0, \sigma]}|\phi(s)|_{\boldsymbol{X}}$, then $|u(t, \sigma, \phi ; p)|_{\boldsymbol{X}}<\varepsilon\left(\operatorname{resp} .|v(t, \sigma, \phi ; p)|_{\boldsymbol{X}}<\varepsilon\right)$ for $t \geq \sigma$.

Definition 2.2. The zero solution of $(\mathrm{E})$ (resp. $\left(\mathrm{E}_{\infty}\right)$ ) is said to be uniformly asymptotically stable (in short, UAS) if and only if for any $\varepsilon>0$, there exists a $\delta(\varepsilon)>0$ such that for any $(\sigma, \phi) \in \boldsymbol{R}^{+} \times B C([0, \sigma] ; \boldsymbol{X})$ (resp. $(\sigma, \phi) \in \boldsymbol{R} \times B C((-\infty, \sigma] ; \boldsymbol{X}))$, if $\|\phi\|_{[0, \sigma]}<\delta(\varepsilon) \quad\left(\right.$ resp. $\left.\|\phi\|_{(-\infty, \sigma]}<\delta(\varepsilon)\right)$, then $|u(t, \sigma, \phi ; 0)|_{X}<\varepsilon$ (resp. $\left.|v(t, \sigma, \phi ; 0)|_{X}<\varepsilon\right)$ for $t \geq \sigma$, in addition, there is a $\delta_{0}>0$ such that for each $\varepsilon^{\prime}>0$ there exists a $l\left(\varepsilon^{\prime}\right)>0$ such that $\|\phi\|_{[0, \sigma]}<\delta_{0}$ (resp. $\left.\|\phi\|_{(-\infty, \sigma]}<\delta_{0}\right)$ implies $|u(t, \sigma, \phi ; 0)|_{X}<\varepsilon^{\prime}\left(\right.$ resp. $\left.|v(t, \sigma, \phi ; 0)|_{X}<\varepsilon^{\prime}\right)$ for $t \geq$ $\sigma+l\left(\varepsilon^{\prime}\right)$.

In addition to the conditions on (E) such as almost periodicity of $B(t, s)$ and so on, we always assume the following two conditions without stating explicitly:

(H1) $\sup _{t \in \boldsymbol{R}} \int_{-\infty}^{t}\|B(t, s)\| d s=: M^{*}<\infty$.

(H2) For any $\eta>0$, there exists an $S(\eta)>0$ such that

$$
\sup _{t \in \boldsymbol{R}} \int_{-\infty}^{t-S(\eta)}\|B(t, s)\| d s<\eta \text {. }
$$

In case that $B(t, s)$ is of convolution type, that is, $B(t, s) \equiv B(t-s)$, Conditions $(\mathrm{H} 1)$ and $(\mathrm{H} 2)$ are equivalent to the integrability of $B(t)$ on $[0, \infty)$, that is, $\int_{0}^{\infty}\|B(t)\| d t<\infty$.

The following proposition is given by the slight modification of Theorems 3.11 and 3.15 in [13]. 
Proposition 2.3. The following four statements are mutually equivalent:

i) The zero solution of $(\mathrm{E})$ is $T S$.

ii) The zero solution of $\left(\mathrm{E}_{\infty}\right)$ is $T S$.

iii) The zero solution of (E) is $U A S$.

iv) The zero solution of $\left(\mathrm{E}_{\infty}\right)$ is $U A S$.

Next, for Equation (E) we will define the resolvent operator, and establish a representation formula for the solutions of the perturbed equations of $(E)$. It is straightforward to certify that there exists an $R(t, \alpha)$ which is a bounded linear operator on $\boldsymbol{X}$ such that $R(t, \alpha)$ is strongly continuous with $t \geq \alpha$,

$$
R(t, \alpha)=0 \quad \text { if } t<\alpha
$$

and

$$
R(t, \alpha) x=T(t-\alpha) x+\int_{\alpha}^{t} T(t-s)\left(\int_{\alpha}^{s} B(s, \tau) R(\tau, \alpha) x d \tau\right) d s, \quad(t \geq \alpha, x \in \boldsymbol{X}) .
$$

Clearly, $R(t, t)=I$, the identy operator. We call $R(t, \alpha)$ the resolvent operator of $(\mathrm{E})$.

For $p \in B C(\boldsymbol{R} ; \boldsymbol{X}), x \in \boldsymbol{X}$ and $\sigma \in \boldsymbol{R}$, put

$$
u(\cdot)=u(\cdot, \sigma, x ; p),
$$

where $u$ is a solution of

$$
\dot{u}(t)=A u(t)+\int_{\sigma}^{t} B(t, s) u(s) d s+p(t), \quad t \geq \sigma,
$$

with $u(\sigma)=x$, that is,

$$
u(t)=T(t-\sigma) x+\int_{\sigma}^{t} T(t-\tau)\left(\int_{\sigma}^{\tau} B(\tau, s) u(s) d s+p(\tau)\right) d \tau, \quad t \geq \sigma .
$$

Since

$$
R(t, \sigma) x=T(t-\sigma) x+\int_{\sigma}^{t} T(t-\tau)\left(\int_{\sigma}^{\tau} B(\tau, s) R(s, \sigma) x d s\right) d \tau, \quad t \geq \sigma
$$

we have

$$
R(t, \sigma) x=u(t, \sigma, x ; 0) \quad t \geq \sigma .
$$

For $t \geq \sigma$, put

$$
z(t)=\int_{\sigma}^{t} R(t, s) p(s) d s
$$


Since

$$
\begin{aligned}
z(t)-\int_{\sigma}^{t} T(t-s) p(s) d s & =\int_{\sigma}^{t}(R(t, r) p(r)-T(t-r) p(r)) d r \\
& =\int_{\sigma}^{t}\left(\int_{r}^{t} T(t-s)\left(\int_{r}^{s} B(s, \tau) R(\tau, r) p(r) d \tau\right) d s\right) d r \\
& =\int_{\sigma}^{t} T(t-s)\left(\int_{\sigma}^{s}\left(\int_{r}^{s} B(s, \tau) R(\tau, r) p(r) d \tau\right) d r\right) d s \\
& =\int_{\sigma}^{t} T(t-s)\left(\int_{\sigma}^{s} B(s, \tau)\left(\int_{\sigma}^{\tau} R(\tau, r) p(r) d r\right) d \tau\right) d s \\
& =\int_{\sigma}^{t} T(t-s)\left(\int_{\sigma}^{s} B(s, \tau) z(\tau) d \tau\right) d s
\end{aligned}
$$

we have

$$
\left.z(t)=\int_{0}^{t} T(t-s)\left(\int_{\sigma}^{s} B(s, \tau) z(\tau) d \tau\right) d \tau+p(s)\right) d s
$$

Thus

$$
u(t, \sigma, 0 ; p)=\int_{\sigma}^{t} R(t, s) p(s) d s \quad \text { for } t \geq \sigma
$$

Therefore we have the following result (the variation of parameters formula) (cf. $[5,6,21])$.

Proposition 2.4. For any $(\sigma, x) \in \boldsymbol{R} \times \boldsymbol{X}$ and any $p \in B C([\sigma, \infty) ; \boldsymbol{X})$, the solution $u(\cdot, \sigma, x ; p)$ of the perturbed equation (1) such that $u(\sigma, \sigma, x ; p)=x$ is given by the formula

$$
u(t, \sigma, x ; p)=R(t, \sigma) x+\int_{\sigma}^{t} R(t, s) p(s) d s, \quad t \geq \sigma .
$$

\section{Stability properties and integrability of the resolvent operator}

Let $(\sigma, \phi) \in \boldsymbol{R}^{+} \times B C([0, \sigma] ; \boldsymbol{X})$ be given, and recall that $u(t):=u(t, \sigma, \phi ; p)$ is a mild solution of

$$
\begin{aligned}
\dot{u}(t) & =A u(t)+\int_{0}^{t} B(t, s) u(s) d s+p(t) \\
& =A u(t)+\int_{\sigma}^{t} B(t, s) u(s) d s+p(t)+\int_{0}^{\sigma} B(t, s) \phi(s) d s, \quad t \geq \sigma .
\end{aligned}
$$


It follows from Proposition 2.4 that

$$
u(t, \sigma, \phi ; p)=R(t, \sigma) \phi(\sigma)+\int_{\sigma}^{t} R(t, \tau)\left(p(\tau)+\int_{0}^{\sigma} B(\tau, s) \phi(s) d s\right) d \tau
$$

for all $t \geq \sigma$, where $R(t, s)$ is the resolvent operator of $(\mathrm{E})$.

Similarly, for any $(\sigma, \psi) \in \boldsymbol{R} \times B C((-\infty, \sigma] ; \boldsymbol{X}), v(t)=v(t, \sigma, \psi ; p)$ is a mild solution of

$$
\begin{aligned}
\dot{v}(t) & =A v(t)+\int_{-\infty}^{t} B(t, s) v(s) d s+p(t) \\
& =A v(t)+\int_{\sigma}^{t} B(t, s) v(s) d s+p(t)+\int_{-\infty}^{\sigma} B(t, s) \psi(s) d s, \quad t \geq \sigma
\end{aligned}
$$

and hence we get

$$
v(t, \sigma, \psi ; p)=R(t, \sigma) \psi(\sigma)+\int_{\sigma}^{t} R(t, \tau)\left(p(\tau)+\int_{-\infty}^{\sigma} B(\tau, s) \psi(s) d s\right) d \tau
$$

for all $t \geq \sigma$.

In this section, applying Relations (2) and (3) we will derive a condition on the resolvent operator $R(t, s)$ which characterizes the UAS property and the TS property of the zero solution of $(E)$ or $\left(E_{\infty}\right)$.

Theorem 3.1. The zero solution of $\left(\mathrm{E}_{\infty}\right)$ is TS (or UAS) if and only if the resolvent operator $R(t, s)$ of $(\mathrm{E})$ satisfies the following condition;

$$
\begin{gathered}
M:=\sup \left\{\|R(t, \sigma)\|+\left|\int_{\sigma}^{t} R(t, \tau) h(\tau) d \tau\right|_{X}:-\infty<\sigma \leq t<\infty,\right. \\
\left.h(\cdot) \in C([\sigma, t] ; \boldsymbol{X}),\|h\|_{[\sigma, t]} \leq 1\right\}<\infty .
\end{gathered}
$$

Consequently, by virtue of Proposition 2.3, Condition (4) is equivalent also to the UAS property (or the TS property) for the zero solution of (E).

Proof. First of all, recall that the UAS property and the TS property for the zero solution of $\left(\mathrm{E}_{\infty}\right)$ or $(\mathrm{E})$ are mutually equivalent, by Proposition 2.3. We first assume that the zero solution of $\left(\mathrm{E}_{\infty}\right)$ is TS, and derive Condition (4). To do this, for any $x \in X,|x|_{X} \leq 1$, let us put

$$
\phi^{n, \sigma}(s)= \begin{cases}{[n(s-\sigma)+1] x,} & \text { for } \sigma-\frac{1}{n} \leq s \leq \sigma \\ 0, & \text { for } s<\sigma-\frac{1}{n}\end{cases}
$$

Then 


$$
\sup _{s \leq \sigma}\left|\phi^{n, \sigma}(s)\right|_{X}=|x|_{X} \leq 1
$$

Thus

$$
\left|v\left(t, \sigma, \phi^{n . \sigma} ; 0\right)\right|_{X} \leq \frac{1}{\delta(1)}
$$

for $t \geq \sigma$. By virtue of (3) we get

$$
\begin{aligned}
|R(t, \sigma) x|_{X} \leq & \left|v\left(t, \sigma, \phi^{n, \sigma} ; 0\right)\right|_{X} \\
& +\left|\int_{\sigma}^{t} R(t, \tau) \int_{\sigma-1 / n}^{\sigma} B(t, s) \phi^{n, \sigma}(s) d s d \tau\right|_{X} \\
\leq & \frac{1}{\delta(1)}+\left|\int_{\sigma}^{t} R(t, \tau) \int_{\sigma-1 / n}^{\sigma} B(t, s) \phi^{n, \sigma}(s) d s d \tau\right|_{X}
\end{aligned}
$$

Letting $n \rightarrow \infty$ in the above, we have

$$
|R(t, \sigma) x|_{X} \leq \frac{1}{\delta(1)}
$$

Therefore

$$
\|R(t, \sigma)\| \leq \frac{1}{\delta(1)}
$$

Furthermore, for any $h \in C([\sigma, t] ; \boldsymbol{X}),\|h\|_{[\sigma, t]} \leq 1$, we get

$$
\left|v\left(t, \sigma, \phi^{n, \sigma} ; h\right)\right|_{\boldsymbol{X}} \leq \frac{1}{\delta(1)}
$$

for all $t \geq \sigma$. Therefore

$$
\left|\int_{\sigma}^{t} R(t, \tau) h(\tau) d \tau\right|_{X} \leq\left|v\left(t, \sigma, \phi^{n \cdot \sigma} ; h\right)\right|_{X}+\left|v\left(t, \sigma, \phi^{n, \sigma} ; 0\right)\right|_{X} \leq \frac{2}{\delta(1)} .
$$

Hence Condition (4) is satisfied; indeed, $M$ is a constant which is not greater than $3 / \delta(1)$.

Next, we shall verify the converse implication. By the assumption, it follows that $\|R(t, \sigma)\|+\left|\int_{\sigma}^{t} R(t, \tau) h(\tau) d \tau\right|_{X} \leq M$ for any $-\infty<\sigma \leq t<\infty$ and any $h(\cdot) \in C([\sigma, t] ; \boldsymbol{X})$ with $\|h\|_{[\sigma, t]} \leq 1$, where $M$ is a constant appearing in Condition (4). Assume that $\|\phi\|_{(-\infty, \sigma]} \leq \delta$ and $\|p\|_{[\sigma, \infty]} \leq \delta$. Then (3) implies that 


$$
\begin{aligned}
|v(t, \sigma, \phi ; p)|_{X} & \leq|R(t, \sigma) \phi(\sigma)|_{X}+\left|\int_{\sigma}^{t} R(t, \tau)\left(p(\tau)+\int_{-\infty}^{\sigma} B(\tau, s) \phi(s) d s\right) d \tau\right|_{X} \\
& \leq M\|\phi\|_{(-\infty, \sigma]}+M\|p\|_{[\sigma, t]}+M M^{*}\|\phi\|_{(-\infty, \sigma]} \\
& \leq M\left(2+M^{*}\right) \delta,
\end{aligned}
$$

which shows that the zero solution of $\left(\mathrm{E}_{\infty}\right)$ is TS.

It is an interesting problem to study whether or not a family $\{Q(t, s)\}$ in $L(\boldsymbol{X})$ satisfying the condition (4) always satisfies the integrability of $Q(t, s)$. In fact, in case the dimension $\boldsymbol{X}$ is finite, the problem is solved affirmatively, and consequently the UAS property for the zero solution of $(\mathrm{E})$ can be characterized in terms of the integrability of the resolvent operator $R(t, \sigma)$ of $(\mathrm{E})$, together with the uniform boundedness of $R(t, \sigma)$; for the details, see [10] and [24]. In case the dimension $\boldsymbol{X}$ is infinite, however, the aspect is quite different. Indeed, in the following example, we will obtain a family $\{Q(t, s)\}$ in $L(\boldsymbol{X})$ with $Q(t, s) \equiv$ $Q(t-s, 0)=: Q(t-s)$ which satisfies Condition (4) but $\int_{0}^{\infty}\|Q(\tau)\| d \tau=\infty$.

Example 3.2. Let us consider the Banach space

$$
\begin{aligned}
X & =l^{\infty}\left(\boldsymbol{Z}^{+} ; \boldsymbol{C}\right) \\
& =\left\{x=\left\{x_{k}\right\}_{k=0}^{\infty}\left|x_{k} \in \boldsymbol{C}, k \in \boldsymbol{Z}^{+}:=\{0,1,2, \ldots\},\|x\|=\sup _{k \in \boldsymbol{Z}^{+}}\right| x_{k} \mid<\infty\right\} .
\end{aligned}
$$

For each $m \in \boldsymbol{Z}^{+}$and any $x=\left\{x_{k}\right\}_{k=0}^{\infty}$, we define $P(m) x \in l^{\infty}\left(\boldsymbol{Z}^{+} ; \boldsymbol{C}\right)$ by $[P(m) x](k)=x_{m}$ if $k=m$, and 0 if $k \neq m$. Clearly, $P(m) \in L(\boldsymbol{X})$ and $\|P(m)\|$ $=1$. Let us consider $Q \in C\left(\boldsymbol{R}^{+} ; L(\boldsymbol{X})\right)$ defined by $Q(t)=(1 / 2-t) P(0)$ if $0 \leq$ $t<1 / 2$, and $(1 / 2-|t-m|) P(m)$ if $m-1 / 2 \leq t<m+1 / 2$ for some positive integer $m$. Then $\sup _{t \in \boldsymbol{R}^{+}}\|Q(t)\|=1 / 2$. Moreover, $\int_{m-1 / 2}^{m+1 / 2}\|Q(t)\| d t=1 / 4$, and hence $\int_{0}^{\infty}\|Q(t)\| d t=\infty$. On the one hand, the family $Q(t, s), t \geq s$, defined by $Q(t, s)=Q(t-s)$ satisfies Condition (4). Indeed, if $h(\cdot)=\left\{h_{m}(\cdot)\right\}_{m \in Z^{+}} \in$ $C([0, l] ; \boldsymbol{X})$ with $\sup _{0 \leq t \leq l}\|h(t)\| \leq 1$, then $\left|\int_{0}^{l} Q(t) h(t) d t\right|_{\boldsymbol{X}}=\sup _{m \in \boldsymbol{Z}^{+}}\left|\int_{0}^{l} x_{m}(t) d t\right|$, where $x_{m}(t)$ is given by $x_{m}(t)=(1 / 2-|t-m|) h_{m}(t)$ if $m-1 / 2 \leq t<m+1 / 2$, and 0 if otherwise. Observe that $\left|x_{m}(t)\right| \leq 1 / 2-|t-m|$ if $|t-m| \leq 1 / 2$, and $x_{m}(t)=0$ if otherwise. This observation leads to $\left|\int_{0}^{l} Q(t) h(t) d t\right|_{X} \leq 1 / 4$. Thus Condition (4) is satisfied.

It is natural to ask in connection with Theorem 3.1 if one can derive the integrability of the resolvent operator of (E) from the UAS property in case of infinite dimensional $\boldsymbol{X}$. Notice that the above example has not answered the question. Indeed, we don't know if the function $Q$ established in Example 3.2 is the resolvent operator of some linear homogeneous Volterra intgrodifferential 
equation. Thus, up to now, the authors have not succeeded in answering the question, in general. In the special case that (E) is of convolution type, however, one can deduce the integrability of the resolvent operator of $(\mathrm{E})$. In fact, as shown in the next theorem, the UAS property (or the TS property) of the zero solution for equations of convolution type can be characterized by the integrability of $R(t)$, as well as the invertibility of the operator $\lambda I-A-\hat{B}(\lambda)$ for any $\lambda$ with $\Re \lambda \geq 0$, where $\hat{B}(\lambda)=\int_{0}^{\infty} B(t) e^{-\lambda t} d t$. Here it should be noticed that if $(\mathrm{E})$ is of convolution type, that is, $B(t, s) \equiv B(t-s)$, the resolvent is of convolution type; that is $R(t, \sigma) \equiv R(t-\sigma, 0)=: R(t-\sigma)$. We emphasize that the next theorem is a generalization of some results in [7] and [16] for equations with infinite dimensional $\boldsymbol{X}$.

Theorem 3.3. Let (E) be the equation of convolution type with $B(t, s) \equiv$ $B(t-s)$. Then the following three statements are mutually equivalent:

i) The zero solution of $(\mathrm{E})$ is $U A S$.

ii) For any $\lambda$ with $\Re \lambda \geq 0$, the operator $\lambda I-A-\hat{B}(\lambda)$ is invertible in $L(X)$.

iii) The resolvent of $(\mathrm{E})$ is integrable on $[0, \infty)$.

The proof of the theorem consists of the verification of the implications (i) $\Rightarrow$ (ii) $\Rightarrow$ (iii) $\Rightarrow$ (i).

Proof of $[(\mathrm{i}) \Rightarrow(\mathrm{ii})]$. In order to establish the implication by a contradiction, we assume that the zero solution of (E) is UAS, and that there exists a $\lambda_{0}$ with $\Re \lambda_{0} \geq 0$ such that $\lambda_{0} I-A-\hat{\boldsymbol{B}}\left(\lambda_{0}\right)$ is not invertible in $L(\boldsymbol{X})$. Take an $s \in \rho(A)$. Then

$$
\begin{aligned}
\left(\lambda_{0} I-A-\hat{B}\left(\lambda_{0}\right)\right)(s I-A)^{-1} & =I+\left(\left(\lambda_{0}-s\right) I-\hat{B}\left(\lambda_{0}\right)\right)(s I-A)^{-1} \\
& =I+K
\end{aligned}
$$

or

$$
\lambda_{0} I-A-\hat{B}\left(\lambda_{0}\right)=(I+K)(s I-A),
$$

where $K:=\left(\left(\lambda_{0}-s\right) I-\hat{B}\left(\lambda_{0}\right)\right)(s I-A)^{-1}$. Notice that $K$ is a compact operator because of the compactness of the semigroup $T(t)$. Since $\lambda_{0} I-A-\hat{B}\left(\lambda_{0}\right)$ is not invertible, the operator $I+K$ is not invertible, and hence $I+K$ is not injective by the Riesz-Schauder theorem on compact operators (e.g., [22, Theorem 4.25]). Take a nonzero $\xi \in X$ such that $(I+K) \xi=0$, and set $\mu=$ $(s I-A)^{-1} \xi$. Then $\left(\lambda_{0} I-A-\hat{B}\left(\lambda_{0}\right)\right) \mu=0$, that is,

$$
\lambda_{0} \mu=A \mu+\int_{0}^{\infty} B(t) \mu e^{-\lambda_{0} t} d t
$$

Define a function $v: \boldsymbol{R} \mapsto \boldsymbol{X}$ by $v(t)=e^{\lambda_{0} t} \mu$. Since $\Re \lambda_{0} \geq 0$, it follows that $v_{0} \in B C\left(\boldsymbol{R}^{-} ; \boldsymbol{X}\right)$. Moreover, $v(t)$ satisfies $\left(\mathrm{E}_{\infty}\right)$, because 


$$
\begin{aligned}
\dot{v}(t)=\lambda_{0} e^{\lambda_{0} t} \mu & =e^{\lambda_{0} t}\left(A \mu+\int_{0}^{\infty} B(s) \mu e^{-\lambda_{0} s} d s\right) \\
& =A v(t)+\int_{0}^{\infty} B(s) v(t-s) d s .
\end{aligned}
$$

Recall that the zero solution of $\left(\mathrm{E}_{\infty}\right)$ is UAS by Proposition 2.3. Therefore we must get $\lim _{t \rightarrow \infty}|v(t)|_{X}=0$, which cotradicts the fact that $|v(t)|_{X} \geq|\mu|_{X}>0$ for $t \geq 0$. This contradiction shows that the implication must hold true.

Proof of $[($ ii) $\Rightarrow$ (iii)]. We first assert that

$$
\hat{R}(z)=(z I-A-\hat{B}(z))^{-1}
$$

for all $z$ with sufficiently large $\Re z$. Indeed, applying Gronwall's inequality, one can easily see that $R(t)$ is bounded exponentially; that is, $\sup _{t \geq 0} e^{-\omega t}\|R(t)\| \leq$ $M$ for some positive constants $M$ and $\omega$. Here we may assume that $\sup _{t \geq 0} e^{-\omega t}\|T(t)\|<\infty$, and hence one can consider the Laplace transform $\hat{T}(z)$ when $\Re z>\omega$. Indeed, it follows that $\hat{T}(z)=(z I-A)^{-1}$ if $\Re z>\omega$. Let $x$ be any element in $\boldsymbol{X}$. Recall that

$$
R(t) x=T(t) x+\int_{0}^{t} T(t-s)\left(\int_{0}^{s} B(s-\tau) R(\tau) x d \tau\right) d s, \quad \forall t \geq 0 .
$$

Considering the Laplace transform of both sides in the above equation, we get

$$
\begin{aligned}
\hat{R}(z) x & =\hat{T}(z) x+\hat{T}(z) \hat{B}(z) \hat{R}(z) x \\
& =(z I-A)^{-1}(x+\hat{B}(z) \hat{R}(z) x),
\end{aligned}
$$

or

$$
(z I-A-\hat{B}(z)) \hat{R}(z) x=x
$$

for $\Re z>\omega$. Notice that the operator $z I-A-\hat{B}(z)$ is invertible by the assumption in (ii). Therefore it follows that $\hat{R}(z) x=(z I-A-\hat{B}(z))^{-1} x$ for any $x \in \boldsymbol{X}$ and $\Re z>\omega$. Thus we get $\hat{R}(z)=(z I-A-\hat{B}(z))^{-1}$ for all $z$ with $\Re z>\omega$, as required.

Now we will establish the integrability of $R$ on $\boldsymbol{R}^{+}$by modifying the method which was employed in [6, Theorem 3.7] and [5, Theorem 5]. Let us take a constant $\omega_{0}$ with $\omega_{0}>\omega$, where $\omega$ is the constant which has appeared in the preceding paragraph. Define $e(t)=T(t) e^{-\omega_{0} t}$ and $D(t)=\int_{0}^{t} B(t-s) e(s) d s+$ $\omega_{0} e(t)$ for $t \geq 0$. Since the semigroup $\{T(t)\}_{t \geq 0}$ is compact, it follows from [19, Theorem 3.2] that $T(t)$ is continuous in $t>0$ as an $L(X)$-valued function, and hence the function $e: \boldsymbol{R}^{+} \mapsto L(\boldsymbol{X})$ is integrable by virtue of the inequality 
$\|e(t)\| \leq\left(\sup _{\tau \geq 0} e^{-\omega \tau}\|T(\tau)\|\right) e^{-\left(\omega_{0}-\omega\right) t}$. Therefore the function $D: \boldsymbol{R}^{+} \mapsto L(\boldsymbol{X})$ is integrable. In what follows, we will verify that for any $z$ with $\Re z \geq 0$, $(I-\hat{D}(z))^{-1}$ is invertible in $L(\boldsymbol{X})$ and

$$
\hat{e}(z)(I-\hat{D}(z))^{-1}=(z I-A-\hat{B}(z))^{-1} .
$$

Indeed, the Laplace transform $\hat{e}(z)$ is given by $\hat{e}(z)=\left(\left(z+\omega_{0}\right) I-A\right)^{-1}$, and hence we get $\hat{D}(z)=\left(\hat{B}(z)+\omega_{0}\right)\left(\left(z+\omega_{0}\right) I-A\right)^{-1}$. Observe that

$$
\begin{aligned}
z I-A-\hat{\boldsymbol{B}}(z) & =\left(\omega_{0}+z\right) I-\left(A+\hat{\boldsymbol{B}}(z)+\omega_{0} I\right) \\
& =(I-\hat{D}(z))\left(\left(\omega_{0}+z\right) I-A\right) .
\end{aligned}
$$

Since the operators $z I-A-\hat{B}(z)$ and $\left(\omega_{0}+z\right) I-A$ are bijective from $D(A)$ into $\boldsymbol{X}$, the operator $I-\hat{D}(z): X \rightarrow X$ is bijective. Hence $I-\hat{D}(z)$ is invertible in $L(\boldsymbol{X})$ with $(I-\hat{D}(z))^{-1}=\left(\left(\omega_{0}+z\right) I-A\right)(z I-A-\hat{B}(z))^{-1}$, which yields Relation (5).

Since $I-\hat{D}(z)$ is invertible for each $z$ with $\Re z \geq 0$, by [21, Theorem 0.7] there exists an integrable $Q: \boldsymbol{R}^{+} \rightarrow L(\boldsymbol{X})$ such that $Q(t)=D(t)+$ $\int_{0}^{t} D(t-\tau) Q(\tau) d \tau$ on $\boldsymbol{R}^{+}$. Observe that $\hat{Q}(z)(I-\hat{D}(z))=\hat{D}(z)$ or $\hat{Q}(z)=$ $\hat{D}(z)(I-\hat{D}(z))^{-1}$. Hence $I+\hat{Q}(z)=(I-\hat{D}(z))^{-1}, \quad$ and $\quad \hat{e}(z)(I+\hat{Q}(z))=$ $\hat{e}(z)(I-\hat{D}(z))^{-1}=(z I-A-\hat{B}(z))^{-1}$ by (5). Set $S(t)=e(t)+\int_{0}^{t} e(t-s) Q(s) d s$ for $t \geq 0$. Then $S$ is integrable on $\boldsymbol{R}^{+}$, and $\hat{S}(z)=(z I-A-\hat{B}(z))^{-1}=\hat{R}(z)$ for all $z$ with sufficiently large $\Re z$. By the uniqueness of Laplace transform, we get $R \equiv S$, which proves the integrability of $R$.

Proof of $[($ iii $) \Rightarrow(\mathrm{i})]$. Assume that $\int_{0}^{\infty}\|R(t)\| d t<\infty$. We will first certify that $\|R(t)\|$ is bounded on $[0, \infty)$. To do this, we choose positive constants $M$ and $\omega$ satisfying $\sup _{t \geq 0} e^{-\omega t}\|T(t)\| \leq M$, and set $S(t)=T(t) e^{-\omega t}$. Then $\{S(t)\}_{t \geq 0}$ is a uniformly bounded $C_{0}$ semigroup with the generator $A-\omega I$. For each $x \in \boldsymbol{X}, u(t):=R(t) x$ is a mild solution of the equation $\dot{u}=A u+g(t)$, where $g(t):=\int_{0}^{t} B(t-s) u(s) d s$. Then $u$ is a mild solution of the equation $\dot{u}=(A-\omega I) u+h(t)$, where $h(t):=g(t)+\omega u(t)$; hence it follows that $u(t)=$ $S(t) u(0)+\int_{0}^{t} S(t-s) h(s) d s$, or

$$
R(t) x=S(t) x+\omega \int_{0}^{t} S(t-s) R(s) x d s+\int_{0}^{t} S(t-s)\left(\int_{0}^{s} B(s-\tau) R(\tau) x d \tau\right) d s .
$$

Indeed, this claim can easily be checked if $u$ is smooth. Also, for $u$ which is not smooth, one can certify the claim by considering a sequence of smooth functions which approximate to $u$. Since $\sup _{t \geq 0}\|S(t)\| \leq M$, we get

$$
\|R(t) x\| \leq M\|x\|\left(1+\omega \int_{0}^{t}\|R(s)\| d s+\int_{0}^{t}\left(\int_{0}^{s}\|B(s-\tau)\|\|R(\tau)\| d \tau\right) d s\right),
$$


which yields the boundedness of $\|R(t)\|$ on $[0, \infty)$, because of

$$
\sup _{t \geq 0}\|R(t)\| \leq M_{1}:=M\left(1+\omega \int_{0}^{\infty}\|R(s)\| d s+\int_{0}^{\infty}\|B(\tau)\| d \tau \int_{0}^{\infty}\|R(s)\| d s\right)<\infty .
$$

Assume that $\|\phi\|_{[0, \sigma]} \leq \delta$ and $\|p\|_{[\sigma, \infty)} \leq \delta$. Then it follows from Relation (2) that

$$
|u(t, \sigma, \phi ; p)|_{\boldsymbol{X}} \leq \delta\left(M_{1}+\int_{0}^{\infty}\|R(s)\| d s+\int_{0}^{\infty}\|B(\tau)\| d \tau \int_{0}^{\infty}\|R(s)\| d s\right),
$$

which shows that the zero solution of (E) is TS. Therefore, the zero solution of (E) is UAS by Proposition 2.3, as required.

\section{Uniform asymptotic stability and $R$-bounded solutions}

In this section, we will study the existence of bounded solutions for perturbed equations

$$
\frac{d u(t)}{d t}=A u(t)+\int_{0}^{t} B(t, s) u(s) d s+p(t), \quad t \in \boldsymbol{R}^{+},
$$

and

$\left(\mathbf{P}_{\infty}\right) \quad \frac{d v(t)}{d t}=A v(t)+\int_{-\infty}^{t} B(t, s) v(s) d s+p(t), \quad t \in \boldsymbol{R}$,

under the stability assumptions on the equations

$$
\frac{d u(t)}{d t}=A u(t)+\int_{0}^{t} B(t, s) u(s) d s, \quad t \in \boldsymbol{R}^{+},
$$

and

$$
\frac{d v(t)}{d t}=A v(t)+\int_{-\infty}^{t} B(t, s) v(s) d s, \quad t \in \boldsymbol{R} .
$$

In the following, we call any $v \in B C(\boldsymbol{R} ; \boldsymbol{X})$ an $\boldsymbol{R}$-bounded solution of $\left(\mathrm{P}_{\infty}\right)$ if $v$ is a (mild) solution of $\left(\mathrm{P}_{\infty}\right)$ on $[\sigma, \infty)$ for any $\sigma \in \boldsymbol{R}$.

Theorem 4.1. Assume that the zero solution of $(\mathrm{E})$ is $U A S$. For any $p \in$ $B C(\boldsymbol{R} ; \boldsymbol{X})$, there exists one and only one $\boldsymbol{R}$-bounded solution of $\left(\mathrm{P}_{\infty}\right)$.

Proof. For $n=1,2,3, \ldots$, put

$$
v^{n}(t)=v\left(t,-n, \overline{0} ; \frac{\delta(1)}{2\|p\|_{\infty}} p\right), \quad t \in \boldsymbol{R},
$$


where $\overline{0}(t)=0, t \in \boldsymbol{R}^{-}$and $\delta(\cdot)$ is the one given for the TS of the zero solution of $\left(\mathrm{E}_{\infty}\right)$. Since

$$
\left(v^{n}\right)_{-n}=\overline{0},
$$

the TS of the zero solution of $\left(\mathrm{E}_{\infty}\right)$ (which is given in Proposition 2.3) implies

$$
\left|v^{n}(t)\right|_{\boldsymbol{X}} \leq 1
$$

and

$$
v^{n}(t)=T(t+n) v^{n}(-n)+\int_{-n}^{t} T(t-s)\left(\int_{-\infty}^{s} B(s, \tau) v^{n}(\tau) d \tau+\frac{\delta(1)}{2\|p\|_{\infty}} p(s)\right) d s
$$

for $t \geq-n$. By using the same arguments as in the proof of Lemma 2 in [11], we can see that the set $\left\{v^{n}(t) \mid t \in \boldsymbol{R}, n=1,2,3, \ldots\right\}$ is relatively compact in $\boldsymbol{X}$ and that the family $\left\{v^{n}(t)\right\}$ is uniformly equicontinuous in $t \in \boldsymbol{R}$. Hence we may assume

$$
v^{n}(t) \rightarrow v(t) \quad \text { as } n \rightarrow \infty
$$

compactly on $\boldsymbol{R}$, taking a subsequence if necessarily. Clearly, $\|v\|_{\infty} \leq 1$. For any $\sigma \in \boldsymbol{R}$, we select an $n$ which satisfies $\sigma \geq-n$. Then we have for $t \geq \sigma$

$$
v^{n}(t)=T(t-\sigma) v^{n}(\sigma)+\int_{\sigma}^{t} T(t-s)\left(\int_{-\infty}^{s} B(s, \tau) v^{n}(\tau) d \tau+\frac{\delta(1)}{2\|p\|_{\infty}} p(s)\right) d s,
$$

and $v^{n}(t)$ tends to

$$
v(t)=T(t-\sigma) v(\sigma)+\int_{\sigma}^{t} T(t-s)\left(\int_{-\infty}^{s} B(s, \tau) v(\tau) d \tau+\frac{\delta(1)}{2\|p\|_{\infty}} p(s)\right) d s
$$

as $n \rightarrow \infty$. Thus, $v(t)$ is an $\boldsymbol{R}$-bounded solution of

$$
\frac{d v(t)}{d t}=A v(t)+\int_{-\infty}^{t} B(t, s) v(s) d s+\frac{\delta(1)}{2\|p\|_{\infty}} p(t), \quad t \in \boldsymbol{R} .
$$

Therfore,

$$
\frac{\delta(1)}{2\|p\|_{\infty}} v(t)
$$

is an $\boldsymbol{R}$-bounded solution of $\left(\mathbf{P}_{\infty}\right)$.

Next, we shall show the uniqueness of $\boldsymbol{R}$-bounded solutions of $\left(\mathrm{E}_{\infty}\right)$. Let $v(t)$ and $w(t)$ be $\boldsymbol{R}$-bounded solutions of $\left(\mathbf{P}_{\infty}\right)$, and put $\bar{v}(t)=v(t)-w(t)$. Then $\bar{v}(t)$ is the $\boldsymbol{R}$-bounded solution of $\left(\mathbf{P}_{\infty}\right)$. Assume $v\left(t_{1}\right) \neq w\left(t_{1}\right)$ for some $t_{1} \in \boldsymbol{R}$. Then

$$
\bar{w}(t):=\frac{\delta_{0}}{2\|\bar{v}\|_{\infty}} \bar{v}(t)
$$


is an $\boldsymbol{R}$-bounded solution of $\left(\mathrm{E}_{\infty}\right)$, where $\delta_{0}$ is the one given for UAS of the zero solution of $\left(\mathrm{E}_{\infty}\right)$. Since

$$
\left\|\bar{w}_{t_{0}}\right\|_{B C} \leq \frac{\delta_{0}}{2}<\delta_{0}, \quad \text { for all } t_{0} \in \boldsymbol{R}
$$

the UAS of the zero solution of $\left(\mathrm{E}_{\infty}\right)$ (which is given in Proposition 2.3) implies that for any $\varepsilon>0$, there exists a $T(\varepsilon)>0$ such that

$$
|\bar{w}(t)|_{X}<\varepsilon \quad \text { for all } t \geq t_{0}+T(\varepsilon) .
$$

Since $t_{0}$ is arbitrary,

$$
|\bar{w}(t)|_{\boldsymbol{X}}<\varepsilon \quad \text { for all } t \in \boldsymbol{R},
$$

which implies

$$
|\bar{w}(t)|_{\boldsymbol{X}}=0 \quad \text { for all } t \in \boldsymbol{R}
$$

that is,

$$
\bar{v}(t)=0 \quad \text { for all } t \in \boldsymbol{R},
$$

which is a contradiction.

In the next theorem, we will derive a representation of the bounded solution ensured in Theorem 4.1 by means of the resolvent operator.

Theorem 4.2. Assume that the zero solution of $(\mathrm{E})$ is $U A S$. Then, for any $p \in B C(\boldsymbol{R} ; \boldsymbol{X})$ the limit

$$
\lim _{\sigma \rightarrow-\infty} \int_{\sigma}^{t} R(t, s) p(s) d s
$$

converges compactly on $\boldsymbol{R}$. Furthermore,

$$
\lim _{\sigma \rightarrow-\infty} \int_{\sigma}^{t} R(t, s) p(s) d s=: \int_{-\infty}^{t} R(t, s) p(s) d s
$$

is the unique $\boldsymbol{R}$-bounded solution of $\left(\mathrm{P}_{\infty}\right)$.

Proof. By Theorem 4.1, $\left(\mathrm{P}_{\infty}\right)$ has a unique $\boldsymbol{R}$-bounded solution, say $v(t)$. Let $\left\{\sigma_{n}\right\}$ be any sequence such that $\lim _{n \rightarrow \infty} \sigma_{n}=-\infty$. By using the same arguments as in the proof of Theorem 4.1, one can see that

$$
v^{n}(t):=v\left(t, \sigma_{n}, 0 ; p\right)=\int_{\sigma_{n}}^{t} R(t, s) p(s) d s
$$

converges to an $\boldsymbol{R}$-bounded solution of $\left(\mathrm{P}_{\infty}\right)$, compactly on $\boldsymbol{R}$. Thus 


$$
\lim _{n \rightarrow \infty} \int_{\sigma_{n}}^{t} R(t, s) p(s) d s=v(t) .
$$

Since $\left\{\sigma_{n}\right\}$ is any sequence such that $\lim _{n \rightarrow \infty} \sigma_{n}=-\infty$, the above observation

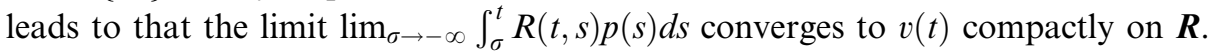

\section{Asymptotically almost periodic solutions}

A function $p \in B C(\boldsymbol{R} ; \boldsymbol{X})$ is said to be asymptotically almost periodic, if

$$
p(t)=q(t)+r(t)
$$

where $q(t)$ is an almost periodic function and $\lim _{t \rightarrow \infty} r(t)=0$. Observe that the almost periodic function $q$ in the above is uniquely determined by the given asymptotically almost periodic function $p$. In what follows, we call $q$ the almost periodic part of $p$, and denote by $q=p_{A P}$.

In this section, we will study the existence of asymptotically almost periodic solutions of $\left(\mathrm{P}_{\infty}\right)$ with asymptotically almost periodic forcing term $p$.

Theorem 5.1. Assume that the zero solution of $(\mathrm{E})$ is $U A S$ and that $p \in$ $B C(\boldsymbol{R} ; \boldsymbol{X})$ is asymptotically almost periodic. Then all solutions of Equations $(\mathrm{P})$ and $\left(\mathbf{P}_{\infty}\right)$ are asymptotically almost periodic, and their almost periodic parts are commonly given by

$$
\int_{-\infty}^{t} R(t, \tau) p_{A P}(\tau) d \tau
$$

Before proving this theorem we prepare the following two lemmas.

Lemma 5.2. Assume that the zero solution of $(\mathrm{E})$ is UAS. For any $r \in$ $B C(\boldsymbol{R} ; \boldsymbol{X})$ such that $\lim _{t \rightarrow \infty} r(t)=0$, we get

$$
\lim _{t \rightarrow \infty} \int_{-\infty}^{t} R(t, s) r(s) d s=0 .
$$

Proof. By Theorem 4.2, $z(t)=\int_{-\infty}^{t} R(t, s) r(s) d s$ is the unique $\boldsymbol{R}$-bounded solution of

$$
\frac{d u(t)}{d t}=A u(t)+\int_{-\infty}^{t} B(t, s) u(s) d s+r(t)
$$

Let $\left\{\tau_{n}\right\}$ be any sequence such that $\lim _{n \rightarrow \infty} \tau_{n}=\infty$, and put

$$
z^{n}(t)=z\left(t+\tau_{n}\right)
$$


By using the same arguments as in the proof of Theorem 4.1, we may assume that the sequence $\left\{z^{n}(t)\right\}$ converges to some function, say $u(t)$, compactly on $\boldsymbol{R}$. Since

$$
\begin{aligned}
\frac{d}{d t} z^{n}(t) & =\frac{d}{d t} z\left(t+\tau_{n}\right) \\
& =A z\left(t+\tau_{n}\right)+\int_{-\infty}^{t+\tau_{n}} B\left(t+\tau_{n}, s\right) z(s) d s+r\left(t+\tau_{n}\right) \\
& =A z^{n}(t)+\int_{-\infty}^{0} B\left(t+\tau_{n}, t+\tau_{n}+s\right) z^{n}(t+s) d s+r\left(t+\tau_{n}\right),
\end{aligned}
$$

$u(t)$ is an $\boldsymbol{R}$-bounded solution of

$$
\frac{d u(t)}{d t}=A u(t)+\int_{-\infty}^{t} C(t, s) u(s) d s,
$$

where $C(t, s)$ is an almost periodic function in the hull of $B(t, s)$. Since the zero solution of $\left(\mathrm{E}_{\infty}\right)$ is UAS by Proposition 2.3 , by repeating the argument in the proof of [13, Theorem 3.11] one can conclude that the zero solution of (6) which is a limiting equation of $\left(\mathrm{E}_{\infty}\right)$ is UAS. In particular, the $\boldsymbol{R}$-bounded solution of (6) must be trivial, and hence $u(t) \equiv 0$. Therefore it follows that $\lim _{n \rightarrow \infty} z^{n}(t)=0$ or $\lim _{n \rightarrow \infty} z\left(t+\tau_{n}\right)=0$. Since $\left\{\tau_{n}\right\}$ is any sequence such that $\lim _{n \rightarrow \infty} \tau_{n}=\infty$, we have $\lim _{t \rightarrow \infty} z(t)=0$, as required.

Lemma 5.3. Assume that the zero solution of (E) is UAS and that $p \in$ $B C(\boldsymbol{R} ; \boldsymbol{X})$ is asymptotically almost periodic. Then $\left(\mathrm{P}_{\infty}\right)$ has a unique $\boldsymbol{R}$-bounded solution, which is asymptotically almost periodic and its almost periodic part is given by

$$
\int_{-\infty}^{t} R(t, \tau) p_{A P}(\tau) d \tau
$$

Proof. By Theorem 4.1, $\left(\mathrm{P}_{\infty}\right)$ has a unique $\boldsymbol{R}$-bounded solution

$$
\int_{-\infty}^{t} R(t, \tau) p(\tau) d \tau=\int_{-\infty}^{t} R(t, \tau) q(\tau) d \tau+\int_{-\infty}^{t} R(t, \tau) r(\tau) d \tau
$$

where $q=p_{A P}$. Since $\lim _{t \rightarrow \infty} \int_{-\infty}^{t} R(t, \tau) r(\tau) d \tau=0$ by Lemma 5.2, it suffices only to certify that the function $z(t):=\int_{-\infty}^{t} R(t, \tau) q(\tau) d \tau$ is almost periodic. In fact, $z(t)$ is the unique $\boldsymbol{R}$-bounded solution of

$$
\frac{d v(t)}{d t}=A v(t)+\int_{-\infty}^{t} B(t, s) v(s) d s+q(t)
$$


and

$$
|z(t)|_{X} \leq M\|q\|_{\infty}, \quad \text { for all } t \in \boldsymbol{R},
$$

because

$$
\left|\int_{\sigma}^{t} R(t, \tau) q(\tau) d \tau\right|_{\boldsymbol{X}} \leq M\|q\|_{[\sigma, t]} \leq M\|q\|_{\infty},
$$

for any $\sigma \leq t$, where $M$ is the number appearing in Condition (4). By using standard arguments, one can see that every equation of the hull of Equation (7) has one and only one $\boldsymbol{R}$-bounded solution which is in the hull of $z(t)$. Therefore, $z(t)$ is almost periodic by Corollary 3.1 in [13].

We are now in a position to prove Theorem 5.1.

Proof. Let $v(t)$ be any solution of $\left(\mathrm{P}_{\infty}\right)$ and $z(t)=\int_{-\infty}^{t} R(t, s) p(s) d s$ be the unique $\boldsymbol{R}$-bounded and asymptotic almost periodic solution of $\left(\mathrm{P}_{\infty}\right)$ ensured by Lemma 5.3. Put $w(t)=v(t)-z(t)$. Then $w(t)$ is a solution of $\left(\mathrm{E}_{\infty}\right)$ defined on $[\sigma, \infty)$, and $\lim _{t \rightarrow \infty} w(t)=0$ by the UAS property of the zero solution of $\left(\mathrm{E}_{\infty}\right)$. Therefore, it follows from Lemma 5.3 that

$$
\lim _{t \rightarrow \infty}\left(v(t)-\int_{-\infty}^{t} R(t, s) p_{A P}(s) d s\right)=\lim _{t \rightarrow \infty}\left(w(t)+\int_{-\infty}^{t} R(t, s) r(s) d s\right)=0,
$$

where $r:=p-p_{A P}$. Thus $v(t)$ is asymptotically almost periodic and its almost periodic part is given by $\int_{-\infty}^{t} R(t, \tau) p_{A P}(\tau) d \tau$.

Next, let $u(t)$ be a solution of $(\mathrm{P})$ defined on $[\sigma, \infty), \sigma \geq 0$. Put

$$
u(t)=u(0), \quad t<0 .
$$

For $t \geq \sigma$, we get

$$
\begin{aligned}
\dot{u}(t) & =A u(t)+\int_{0}^{t} B(t, s) u(s) d s+p(t) \\
& =A u(t)+\int_{-\infty}^{t} B(t, s) u(s) d s+p(t)-\left(\int_{-\infty}^{0} B(t, s) d s\right) u(0) .
\end{aligned}
$$

Set

$$
\bar{p}(t)= \begin{cases}-\left(\int_{-\infty}^{0} B(t, s) d s\right) u(0), & \text { for } t \geq \sigma \\ -\left(\int_{-\infty}^{0} B(\sigma, s) d s\right) u(0), & \text { for } t<\sigma .\end{cases}
$$

Then $u(t)$ is a solution of

$$
\dot{v}(t)=A v(t)+\int_{-\infty}^{t} B(t, s) v(s) d s+p(t)+\bar{p}(t)
$$


defined on $[\sigma, \infty)$. Since

$$
\left\|\int_{-\infty}^{0} B(t, s) d s\right\| \leq \int_{-\infty}^{t-S(\varepsilon)}\|B(t, s)\| d s<\varepsilon \quad \text { for } t \geq S(\varepsilon)>\sigma,
$$

we get

$$
\lim _{t \rightarrow \infty}\left(\int_{-\infty}^{0} B(t, s) d s\right) u(0)=0 .
$$

Thus $p+\bar{p} \in B C(\boldsymbol{R} ; \boldsymbol{X})$ is asymptotically almost periodic, and its almost periodic part is $p_{A P}$. Therefore, by Lemma 5.3, $u(t)$ is asymptotically almost periodic and its almost periodic part is given by $\int_{-\infty}^{t} R(t, s) p_{A P}(s) d s$.

For $f \in A P(\boldsymbol{R} ; \boldsymbol{X})$, put

$$
a(f, \lambda)=\lim _{T \rightarrow \infty} \frac{1}{2 T} \int_{T}^{T} e^{-i \lambda t} f(t) d t
$$

The set $\{\lambda \in \boldsymbol{R}: a(f, \lambda) \neq 0\}$ is called the Bohr spectrum of $f$ which will be denoted by $\sigma_{b}(f)$. For details of the Bohr spectrum, see [14]. We denote by $m_{b}(f)$ the module generated by $\sigma_{b}(f)$. An almost periodic function $f$ is called a quasiperiodic function, if $m_{b}(f)$ has a finite integer basis (cf. [14, p. 26 and p. 48]). In what follows, we will give a more precise information on the almost periodic part of asymptotically almost periodic solutions of $\left(\mathrm{P}_{\infty}\right)$ ensured in Theorem 5.1 in connection with the Bohr spectrum, under the periodicity condition on $B(t, s)$ :

(H3) $B(t+\omega, s+\omega) \equiv B(t, s)$ for some constant $\omega>0$.

It is a simple task to see that under Condition (H3), the resolvent operator $R(t, s)$ of $(\mathrm{E})$ is $\omega$-periodic; that is, $R(t+\omega, s+\omega) \equiv R(t, s)$.

Theorem 5.4. Let Conditions $(\mathrm{H} 1)-(\mathrm{H} 3)$ be satisfied, and assume that the zero solution of $(\mathrm{E})$ is $U A S$ and that $p \in B C(\boldsymbol{R} ; \boldsymbol{X})$ is asymptotically almost periodic. Then $\left(\mathrm{P}_{\infty}\right)$ has a unique $\boldsymbol{R}$-bounded solution, which is asymptotically almost periodic and its almost periodic part is given by

$$
\int_{-\infty}^{t} R(t, \tau) p_{A P}(\tau) d \tau
$$

Moreover, the almost periodic part satisfies the relation

$$
\sigma_{b}\left(\int_{-\infty}^{t} R(t, \tau) p_{A P}(\tau) d \tau\right) \subset \sigma_{b}\left(p_{A P}\right)+\frac{2 \pi}{\omega} \boldsymbol{Z} .
$$

Proof. The former part of the theorem has already been established in Theorem 5.1. We will show only the assertion on the Bohr spectrum for the 
almost periodic part $z(t):=\int_{-\infty}^{t} R(t, \tau) q(\tau) d \tau$, where $q:=p_{A P}$. For any $\varepsilon>0$, there exists a

$$
q^{\varepsilon}(t)=\sum_{k=1}^{j} a_{k} e^{i \lambda_{k} t}, \quad \lambda_{k} \in \sigma_{b}(q)
$$

such that

$$
\sup _{t \in \boldsymbol{R}}\left|q(t)-q^{\varepsilon}(t)\right|_{\boldsymbol{X}}<\varepsilon
$$

(see, [14]). Putting

$$
z^{\varepsilon}(t)=\sum_{k=1}^{j} \int_{-\infty}^{t} R(t, \tau) a_{k} e^{i \lambda_{k} \tau} d \tau
$$

we have

$$
\left|z(t)-z^{\varepsilon}(t)\right|_{\boldsymbol{X}} \leq M\left\|q-q^{\varepsilon}\right\|_{\infty}<M \varepsilon \quad \text { for all } t \in \boldsymbol{R},
$$

where $M$ is the number in Condition (4). Put

$$
\phi(t)=\left(\int_{-\infty}^{t} R(t, \tau) a_{k} e^{i \lambda_{k} \tau} d \tau\right) e^{-i \lambda_{k} t} .
$$

Then

$$
\begin{aligned}
\phi(t+\omega) & =\left(\int_{-\infty}^{t+\omega} R(t+\omega, \tau) a_{k} e^{i \lambda_{k} \tau} d \tau\right) e^{-i \lambda_{k}(t+\omega)} \\
& =\left(\int_{-\infty}^{t} R(t+\omega, s+\omega) a_{k} e^{i \lambda_{k}(s+\omega)} d s\right) e^{-i \lambda_{k}(t+\omega)} \\
& =\left(\int_{-\infty}^{t} R(t, s) a_{k} e^{i \lambda_{k} s} d s\right) e^{-i \lambda_{k} t} \\
& =\phi(t),
\end{aligned}
$$

where we used the $\omega$-periodicity of $R(t, s)$. Thus $\phi(t)$ is $\omega$-periodic, and hence $z^{\varepsilon}(t)$ is almost periodic.

Next, we shall establish the relation (8). Since

$$
\sigma_{b}\left(\int_{-\infty}^{t} R(t, \tau) a_{k} e^{i \lambda_{k} \tau} d \tau\right)=\sigma_{b}\left(\phi(t) e^{i \lambda_{k} \tau}\right) \subset\left\{\lambda_{k}\right\}+\frac{2 \pi}{\omega} \boldsymbol{Z} \subset \sigma_{b}(q)+\frac{2 \pi}{\omega} \boldsymbol{Z},
$$

we get

$$
\sigma_{b}\left(z^{\varepsilon}\right) \subset \sigma_{b}(q)+\frac{2 \pi}{\omega} \boldsymbol{Z} .
$$


We assert that

$$
\sigma_{b}(z) \subset \sigma_{b}(q)+\frac{2 \pi}{\omega} \boldsymbol{Z}
$$

Indeed, if the assertion is false, there exists a $\lambda \in \sigma_{b}(z)$, but not in $\sigma_{b}(q)+$ $(2 \pi / \omega) Z$. Since $\lim _{T \rightarrow \infty}(2 T)^{-1} \int_{-T}^{T} e^{-i \lambda t} z(t) d t \neq 0$, there exists $\varepsilon>0$ and $T_{0}>0$ such that for all $T>T_{0},\left|(2 T)^{-1} \int_{-T}^{T} e^{-i \lambda t} z(t) d t\right|_{X} \geq 2 M \varepsilon$. By (9), it follows that

$$
\begin{aligned}
\left|\frac{1}{2 T} \int_{-T}^{T} e^{-i \lambda t} z^{\varepsilon}(t) d t\right|_{X} & =\left|\frac{1}{2 T} \int_{-T}^{T} e^{-i \lambda t}\left(z^{\varepsilon}(t)-z(t)\right) d t+\frac{1}{2 T} \int_{-T}^{T} e^{-i \lambda t} z(t) d t\right|_{\boldsymbol{X}} \\
& \geq\left|\frac{1}{2 T} \int_{-T}^{T} e^{-i \lambda t} z(t) d t\right|_{\boldsymbol{X}}-M \varepsilon \\
& >M \varepsilon .
\end{aligned}
$$

Thus

$$
\lambda \in \sigma_{b}\left(z^{\varepsilon}\right) \subset \sigma_{b}(q)+\frac{2 \pi}{\omega} \boldsymbol{Z}
$$

which is a contradiction. This completes the proof of the theorem.

The following result follows from Theorem 5.4 immediately, because the module of the almost periodic part of the asymptotically almost periodic solution of $\left(\mathrm{P}_{\infty}\right)$ is contained into the module generated by $\sigma_{b}\left(p_{A P}\right)$ and $2 \pi / \omega$, by (8).

Corollary 5.5. Let Conditions $(\mathrm{H} 1)-(\mathrm{H} 3)$ be satisfied, and assume that the zero solution of $(\mathrm{E})$ is $U A S$ and that $p \in B C(\boldsymbol{R} ; \boldsymbol{X})$ is quasiperiodic. Then $\left(\mathrm{P}_{\infty}\right)$ has a unique $\boldsymbol{R}$-bounded solution, and its almost periodic part is given by

$$
\int_{-\infty}^{t} R(t, \tau) p_{A P}(\tau) d \tau
$$

which is quasiperiodic.

Let us consider more general Volterra integrodifferential equations

$$
\dot{u}(t)=A u(t)+\int_{0}^{t}(B(t, s)+E(t, s)) u(s) d s+p(t),
$$

and 


$$
\dot{v}(t)=A v(t)+\int_{-\infty}^{t}(B(t, s)+E(t, s)) v(s) d s+p(t)
$$

where $E(t, s)$ satisfies the following two conditions:

(H4) $\lim _{t \rightarrow \infty}\|E(t, t+s)\|=0$ compactly in $s \in(-\infty, 0]$.

(H5) For any $\eta>0$, there exists an $s(\eta)>0$ such that $\sup _{t \in \boldsymbol{R}} \int_{-\infty}^{t-s(\eta)}\|E(t, s)\| d s$ $<\eta$.

Several results such as Theorems 5.1 and 5.4 hold true for the equations (10) and (11), too. Indeed, we can get the following result which is exacly the same as Theorem 5.4 in the case of $E(t, s) \equiv 0$. The proof of the theorem can be done by using Lemmas 5.2 and 5.3, as in the proof of Theorem 5.1; so, we omit it.

Theorem 5.6. Let Conditions (H1)-(H5) be satisfied, and assume that the zero solution of $(\mathrm{E})$ is $U A S$ and that $p \in B C(\boldsymbol{R} ; \boldsymbol{X})$ is asymptotically almost periodic. Then all solutions of Equations (10) and (11) are asymptotically almost periodic, and their almost periodic parts are given by

$$
\int_{-\infty}^{t} R(t, \tau) p_{A P}(\tau) d \tau
$$

Moreover, the almost periodic part satisfies the relation

$$
\sigma_{b}\left(\int_{-\infty}^{t} R(t, \tau) p_{A P}(\tau) d \tau\right) \subset \sigma_{b}\left(p_{A P}\right)+\frac{2 \pi}{\omega} \boldsymbol{Z} .
$$

Before concluding the paper, we will give an example which shows how our Theorem 4.1 and Theorem 5.1 are applicable.

Example 5.7. Let us consider the following integrodifferential equation with diffusion

$$
\frac{\partial u}{\partial t}(t, x)=\frac{\partial^{2} u}{\partial x^{2}}(t, x)-u(t, x)+\int_{-\infty}^{t} k(t, s, x) u(s, x) d s, \quad t>0,0<x<\pi
$$

In what follows, we shall treat the equation (12) under the Neumann boundary condition

$$
\frac{\partial u}{\partial x}(t, 0)=\frac{\partial u}{\partial x}(t, \pi)=0, \quad t>0
$$

and give a sufficient condition under which the zero solution of (12) is TS.

We assume that $k(t, s, x)$ is a continuous function satisfying $0 \leq k(t, s, x) \leq$ $K(t-s)$ for some continuous function $K(\tau)$ with $\int_{0}^{\infty} K(\tau) d \tau<\infty$. Let us 
consider the Banach space $\boldsymbol{X}=C([0, \pi] ; \boldsymbol{R})$, and define a linear operator $A$ in $\boldsymbol{X}$ by

$$
(A \xi)(x)=\frac{d^{2} \xi}{d x^{2}}(x)-\xi(x), \quad 0<x<\pi
$$

for

$$
\xi \in D(A):=\left\{\xi \in C^{2}[0, \pi]: \xi^{\prime}(0)=\xi^{\prime}(\pi)=0\right\} .
$$

Then the operator $A$ generates a compact semigroup $T(t)$ on $\boldsymbol{X}$, and (12)-(13) is represented as the integrodifferential equation $\left(\mathrm{E}_{\infty}\right)$ on $\boldsymbol{X}$ with

$$
(B(t, s) \xi)(x)=k(t, s, x) \xi(x)
$$

for $\xi \in \boldsymbol{X}$. Obviously, Conditions $(\mathrm{H} 1)$ and $(\mathrm{H} 2)$ are satisfied for this $B(t, s)$. Also, if $k(t, s, x)$ is almost periodic in $t$ uniformly for $(s, x)$, then $B(t, s)$ is almost periodic in $t$ uniformly for $s$. In what follows, we refer to a (mild) solution of $\left(\mathrm{E}_{\infty}\right)$ as a (mild) solution of (12)-(13).

Lemma 5.8. Assume that $\int_{0}^{\infty} K(\tau) d \tau:=a<1$. Then the zero solution of (12)-(13) is TS.

Proof. Let $\sigma \in \boldsymbol{R}$ and $\varepsilon>0$ be given. In order to establish the lemma, we will verify that for any $h \in C([\sigma, \infty) \times[0, \pi] ; \boldsymbol{R})$ with $\sup _{t \geq \sigma, x \in[0, \pi]}|h(t, x)|<$ $(1-a) \varepsilon$, a solution $u(t, x)$ of the equation

$$
\begin{aligned}
\frac{\partial u}{\partial t}(t, x)= & \frac{\partial^{2} u}{\partial x^{2}}(t, x)-u(t, x) \\
& +\int_{-\infty}^{t} k(t, s, x) u(s, x) d s+h(t, x), \quad t>\sigma, 0<x<\pi
\end{aligned}
$$

subject to the condition (13) satisfies $|u(t, x)|<\varepsilon$ for any $(t, x) \in[\sigma, \infty) \times[0, \pi]$ whenever $\sup _{\theta \leq \sigma, x \in[0, \pi]}|u(\theta, x)|<\varepsilon$. Suppose that this assertion is false. Then there is a $\left(t_{1}, x_{1}\right) \in(\sigma, \infty) \times[0, \pi]$ such that $\left|u\left(t_{1}, x_{1}\right)\right|=\varepsilon$ and $|u(t, x)|<\varepsilon$ for any $(t, x) \in\left(-\infty, t_{1}\right) \times[0, \pi]$. Put

$$
p(t, x)=\int_{-\infty}^{t} k(t, s, x) u(s, x) d s+h(t, x), \quad(t, x) \in\left[\sigma, t_{1}\right] \times[0, \pi] .
$$

Then $p \in C\left(\left[\sigma, t_{1}\right] \times[0, \pi] ; \boldsymbol{R}\right)$ with $|p(t, x)| \leq a \varepsilon+\sup _{t \geq \sigma, x \in[0, \pi]}|h(t, x)|<\varepsilon$. Let us choose a constant $\eta(0<\eta<\varepsilon)$ and sequences of functions $\left\{p_{n}\right\} \in C^{1}\left(\left[\sigma, t_{1}\right]\right.$ $\times[0, \pi] ; \boldsymbol{R})$ and $\left\{\phi_{n}\right\} \subset D(A)$ such that $\sup _{n \in N,(t, x) \in\left[\sigma, t_{1}\right] \times[0, \pi]}\left|p_{n}(t, x)\right|<\eta$ with $p_{n}(t, x) \rightarrow p(t, x)$ uniformly on $\left[0, t_{1}\right] \times[0, \pi]$ as $n \rightarrow \infty$, and that $\sup _{n \in N, \in[0, \pi]}$. $\left|\phi_{n}(x)\right|<\eta$ with $\phi_{n}(x) \rightarrow u(\sigma, x)$ uniformly on $[0, \pi]$ as $n \rightarrow \infty$. There exists a (classical) solution $v_{n}(t, x)$ of the initial-boundary value problem 


$$
\begin{aligned}
\frac{\partial v}{\partial t}(t, x) & =\frac{\partial^{2} v}{\partial x^{2}}(t, x)-v(t, x)+p_{n}(t, x), \quad \sigma<t \leq t_{1}, \quad 0<x<\pi, \\
\frac{\partial v}{\partial x}(t, 0) & =\frac{\partial v}{\partial x}(t, \pi)=0, \quad \sigma<t \leq t_{1}, \\
v(0, x) & =\phi_{n}(x), \quad 0<x<\pi .
\end{aligned}
$$

Clearly, $v_{n}(t, x) \rightarrow u(t, x)$ uniformly on $\left[\sigma, t_{1}\right] \times[0, \pi]$. We now assert that

$$
\left|v_{n}(t, x)\right|<\eta \quad \text { on }\left[\sigma, t_{1}\right] \times[0, \pi] .
$$

In order to verify the assertion by a contradiction, we assume that (15) does not hold true. Then there exists a $\left(t_{2}, x_{2}\right) \in\left(\sigma, t_{1}\right] \times[0, \pi]$ such that $\left|v_{n}\left(t_{2}, x_{2}\right)\right|=\eta$ and that $\left|v_{n}(t, x)\right|<\eta$ on $\left[\sigma, t_{2}\right) \times[0, \pi]$. Let us consider the case $v_{n}\left(t_{2}, x_{2}\right)=\eta$, and put $V(t, x)=v_{n}(t, x)-\eta$ for $(t, x) \in\left[\sigma, t_{1}\right] \times[0, \pi]$. Then

$$
\frac{\partial^{2} V}{\partial x^{2}}(t, x)-\frac{\partial V}{\partial t}(t, x)-V(t, x)>0
$$

on $\left(\sigma, t_{1}\right] \times[0, \pi]$, because of

$$
\begin{aligned}
\frac{\partial^{2} V}{\partial x^{2}}(t, x)-\frac{\partial V}{\partial t}(t, x)-V(t, x) & =\frac{\partial^{2} v_{n}}{\partial x^{2}}(t, x)-\frac{\partial v_{n}}{\partial t}(t, x)-v_{n}(t, x)+\eta \\
& =-p_{n}(t, x)+\eta>0 .
\end{aligned}
$$

Therefore, by the strong maximum principle (cf., e.g. [20, Theorem 3.7]) there will arise a contradiction. Indeed, if $x_{2} \in(0, \pi)$, then $\partial^{2} V / \partial x^{2} \leq 0$ and $\partial V / \partial t$ $\geq 0$ and $V=0$, and consequently the left hand side of the above inequality must be nonpositive at $\left(t_{2}, x_{2}\right)$; a contradiction. On the one hand, if $x_{2}=\pi$ (or $x_{2}=0$ ), then the strong maximum principle implies that $\left(\partial v_{n} / \partial x\right)\left(t_{2}, \pi\right)>0$ (or $\left.\left(\partial v_{n} / \partial x\right)\left(t_{2}, 0\right)<0\right)$, a contradiction, because of $\left(\partial v_{n} / \partial x\right)\left(t_{2}, \pi\right)=\left(\partial v_{n} / \partial x\right)\left(t_{2}, 0\right)$ $=0$. Thus, the case $v_{n}\left(t_{2}, x_{2}\right)=\eta$ cannot occur. Similarly, in case of $v_{n}\left(t_{2}, x_{2}\right)$ $=-\eta$, one can derive a contradiction by considering a function $W(t, x):=$ $-\eta-v_{n}(t, x)$ and applying the strong maximum principle. This completes the proof of assertion (15).

Now, let $n \rightarrow \infty$ in assertion (15). Then $|u(t, x)| \leq \eta$ on $\left[\sigma, t_{1}\right] \times[0, \pi]$. In particular, we get $\left|u\left(t_{1}, x_{1}\right)\right| \leq \eta<\varepsilon$, which is a contradiction to $\left|u\left(t_{1}, x_{1}\right)\right|=\varepsilon$, completing the proof of the lemma.

By virtue of Lemma 5.8 and Theorem 4.2 (or Theorem 5.1), one can see that if $\int_{0}^{\infty} K(\tau) d \tau<1$ and $k(t, s, x)$ is almost periodic in $t$ uniformly for $(s, x)$, then the problem (14)-(13) possesses a unique (mild) $\boldsymbol{R}$-bounded solution (or asymptotically almost periodic solution) whenever $h(t, x)$ is bounded on $\boldsymbol{R} \times[0, \pi]$ (or asymptotically almost periodic in $t$ uniformly for $x \in[0, \pi]$ ). 


\section{References}

[1] Buse, C. and Lupulescu, V., Exponential stability of linear and almost periodic systems on Banach spaces, Electron. J. Diff. Eq., 125 (2003), 1-7.

[2 $]$ Chicone, C. and Latushkin, Y., Evolution Semigroups in Dynamical Systems and Differential Equations, Mathematical Surveys and Monographs, 70, American Mathematical Society, Providence, RI, 1999.

[3] Chill, R. and Prüss, J., Asymptotic behaviour of linear evolutionary integral equations, Integral Equations Operator Theory, 39 (2001), 193-213.

[4] Gripenberg, G., On the resolvents of nonconvolution Volterra kernels, Funkcial. Ekvac., 23 (1980), 83-95.

[ 5 ] Gripenberg, G., Asymptotic behaviour of resolvents of abstract Volterra equations, J. Math. Anal. Appl., 122 (1987), 427-438.

[6] Gripenberg, G., Londen, L. O. and Staffans, O. J., Volterra Integral and Functional Equations, Cambridge Univ. Press, Cambridge, 1990.

[7] Grossman, S. I. and Miller, R. K., Nonlinear Volterra integrodifferential systems with $L^{1}$ kernels, J. Differential Equations, 13 (1973), 551-566.

[ 8 ] Henríquez, H. R., Periodic solutions of quasi-linear partial functional differential equations with unbounded delay, Funkcial. Ekvac., 37 (1994), 329-343.

[9] Hino, Y. and Murakami, S., Total stability and uniform asymptotic stability for linear Volterra equations, J. London Math. Soc., 43 (1991), 305-312.

[10] Hino, Y. and Murakami, S., Stability properties of linear Volterra equations, J. Differential Equations, 89 (1991), 121-137.

[11] Hino, Y. and Murakami, S., Limiting equations and some sability properties for asymptotically almost periodic functional differential equations with infinite delay, Tohoku Math. J., 54 (2002), 239-257.

[12] Hino, Y., Murakami, S. and Naito, T., Functional Differential Equations with Infinite Delay, Lecture Notes in Math. 1473, Springer-Verlag, Berlin-New York, 1991.

[13] Hino, Y., Naito, T., Nguyen Van Minh and Shin, J. S., Almost Periodic Solutions of Differential Equations in Banach Spaces, Taylor and Fishers, 2002.

[14] Levitan, B. M. and Zhikov, V. V., Almost Periodic Functions and Differential Equations, Moscow Univ. Publ. Publ. House 1978. English translation by Cambridge University Press 1983.

[15] Lizama, C. and Vergara, V., Uniform stability of resolvent families, Proc. Amer. Math. Soc., 132 (2004), 175-181.

[16] Miller, R. K., Asymptotic stability properties of linear Volterra integrodifferential equations, J. Differential Equations, 10 (1971), 485-506.

[17] Miller, R. K., Linear Volterra integrodifferential equations as semigroups, Funkcial. Ekvac., 17 (1974), 39-55.

[18] Nguyen Van Minh, Räbiger, F. and Schnaubelt, R., Exponential stability, exponential expansiveness, and exponential dichotomy of evolution equations on the half-line, Integral Equations Operator Theory, 32 (1998), 332-353.

[19] Pazy, A., Semigroups of Linear Operators and Applications to Partial Differential Equations, Applied Math. Sci. 44, Springer-Verlag, New York, 1983.

[20] Protter, M. H. and Weinberger, H. F., Maximum Principles in Differential Equations, Springer-Verlag, New York, 1984.

[21] Prüss, J., Evolutionary Integral Equations and Applications, Birkhäuser, Basel, 1993.

[22] Rudin, W., Functional Analysis, McGraw-Hill, New Delhi, 1988. 
[23] Yoshizawa, T., Stability Thery and the Existence of Periodic Solution and Almost Periodic Solutions, Applied Math. Sciences 14, Springer-Verlag, New York, 1975.

[24] Zhang, B., Asymptotic stability criteria and integrability properties of the resolvent of Volterra and functional equations, Funkcial. Ekvac., 40 (1997), 335-351.

\author{
nuna adreso: \\ Yoshiyuki Hino \\ Department of Mathematics and Informatics \\ Chiba University \\ 1-33 Yayoicho, Inageku, Chiba 263-8522 \\ Japan \\ E-mail: hino@math.s.chiba-u.ac.jp \\ Satoru Murakami \\ Department of Applied Mathematics \\ Okayama University of Science \\ 1-1 Ridaicho, Okayama 700-0005 \\ Japan \\ E-mail: murakami@youhei.xmath.ous.ac.jp
}

(Ricevita la 9-an de junio, 2004)

(Reviziita la 26-an de oktobro, 2004) 